\title{
REVUE
}

\section{L'INDUSTRIE LAITIÈRE DANS LE MONDE}

par G. GENIN

Ingénieur E.P.C.

\section{HOLLANDE}

\section{Production laitière}

Actuellement, le nombre de vaches laitières s'élève à 1,5 million, elles fournissent par jour 3.300 .000 gallons de lait. L'industrie laitière est particulièrement florissante et constitue une des richesses du pays. Les exportations de produits laitiers représentent par exemple $10 \%$ des exportations totales hollandaises.

Ce résultat est dû à de nombreuses années d'expérience, à une production par tête de bétail qui atteint les valeurs les plus élevées enregistrées dans le monde, à un excellent outillage des laiteries et à une qualité parfaite des produits laitiers. En 1951, la production des produits laitiers assurée par 500 laiteries et environ 5.000 fermes, s'est élevée à 143.000 tonnes de fromage, 83.000 tonnes de beurre, 179.000 tonnes de lait condensé et 34.000 tonnes de lait en! poudre. Dans la même année, il a été exporté 73.000 tonnes de fromage, 54.000 tonnes de beurre, 175.000 tonnes de lait condensé, 20.000 tonnes de lait en poudre, 2.000 tonnes de poudre de petit-lait, 3.000 tonnes de crème, 3.000 tonnes de caséine et 19.000 tonnes de lait pasteurisé et stérilisé. La valeur de ces exportations s'est élevée à 66 millions de livres.

\section{Organisation de la production}

Dans les laiteries hollandaises, le lait est livré aux laiteries dans la matinée et est complètement traité dès 3 heures de l'après-midi. Après écrémage, il est pasteurisé à $192^{\circ} \mathrm{F}$. et immédiatement refroidi à $48^{\circ} \mathrm{F}$. La crème n'a pas besoin d'être neutralisée, car elle ne subit aucune altération acide. Le beurre fabriqué est uniquement préparé en partant de crème de culture et sa teneur en diacétyle est augmentée par l'emploi d'éveilleurs qui développent la quantité de diacétyle contenu dans le beurre.

Les installations frigorifiques dans les Pays-Bas sont excessivement nombreuses et parfaitement organisées. Les coopératives laitières ont construit d'importants frigidaires qui sont uniquement destinés au stockage des produits laitiers : beurre et fromage. L'industrie laitière est organisée dans le cadre de l'Association laitière des Pays-Bas. L'industrie est soumise à un contrôle d'Etat qui possède différents laboratoires répartis dans le pays et qui 
examine la totalité du beurre fabriqué en vue de sa vente, beurre qui est classé en quatre catégories suivant sa qualité.

\section{La stérilisation $\mathrm{du}$ lait}

En Hollande, le lait destiné à la vente à la consommation est généralement pasteurisé par un traitement d'une durée de 16 secondes à la température de $71^{\circ} \mathrm{C}$. Ce lait ne peut être vendu que s'il présente une réaction négative à la phosphatase. Avec cette méthode de pasteurisation, une bouteille de lait qui accidentellement a été portée pendant deux et même trois jours à 18 ou $20^{\circ}$, est encore parfaitement utilisable et toute la saveur du lait est conservée.

On recherche également actuellement à réaliser des méthodes de stérilisation permettant d'effectuer le traitement sur des quantités importantes de lait. On dispose dans ee but de chambres de stérilisation dites stationnaires, de chambres rotatives ou d'appareils permettant un traitement en continu. On a également réalisé des appareils à fonctionnement continu qui permettent de pasteuriser le lait en bouteille.

\section{Un nouveau dispositif pour le paraffinage du fromage.}

La Société U. P. et Th. Woudstra, spécialisée dans la fabrication de l'outillage laitier vient de mettre au point un nouvel appareil qui permet de recouvrir le fromage d'une mince pellicule de cire. Ce traitement a pour objet d'améliorer les qualités de conservation du produit, d'empêcher son séchage et de s'opposer au développement des moisissures.

L'opération se fait en trempant les fromages dans un bain de cire et en transmettant au fromage, à sa sortie du bain, un mouvement de rotation, de façon à assurer une répartition parfaite de la pellicule de cire. La machine permet de traiter 2.000 fromages d'Edam à l'heure, ou environ 1.000 fromages Gouda.

\section{ALLEMAGNE}

\section{Nouveau type de baratte}

La Société Ahlborn Co. d'Hildesheim vient de construire une nouvelle baratte entièrement métallique dont le tambour est en acier inoxydable ou en acier plaqué par un revêtement d'acier inoxydable. Ce revêtement subit un traitement spécial qui empêche le collage du beurre vers la fin de l'opération lorsqu'on travaille à sec. Cette baratte ne comporte pas de rouleau. 


\section{La congélation du lait}

La question du lait congelé fait l'objet actuellement de nombreuses études en Allemagne occidentale. Tout d'abord, ce procédé permettrait d'assurer la conservation du lait et il constituerait en outre un mode de livraison de ce produit permettant au consommateur de le conserver dans un réfrigérant domestique.

L'application de cette technique présente néanmoins certaines difficultés, en particulier lorsqu'il faut opérer sur de petites rations correspondant à la consommation d'une seule famille. On estime que le problème ne peut être résolu que pour des quantités qui ne doivent pas être inférieures à 5 litres. La question se pose également du transport entre l'usine et le consommateur du lait réfrigéré. On pense donc qu'il s'écoulera encore un certain temps, avant que l'emploi du lait congelé se généralise.

\section{SUISSE}

\section{Nouvelle qualité de poudre de lait}

L'industrie suisse annonce la mise au point d'une nouvelle qualité de poudre de lait qui peut se conserver indéfiniment, aussi bien au Sahara qu'à l'Equateur et que sur les plus hautes montagnes du monde.

Pour utiliser ce produit, il suffit de lui ajouter la quantité d'eau nécessaire et de chauffer le mélange, ce qui peut s'effectuer dans n'importe quel récipient. La mise au point de ce procédé est due à l'Union des Laiteries du Canton de Vaud et le procédé de dessiccation peut être également appliqué au fromage et au vin.

\section{ÉTATS-UNIS}

\section{La guérison des brûlures par le lait}

M. O. M. RIChards, directeur de l'American Dairy Association, a signalé que la récente découverte selon laquelle le lait peut être utilisé avec succès pour le traitement de brûlures sérieuses constitue un fait d'une importance qu'il ne faut pas négliger.

Cette personnalité a signalé que ce nouveau traitement a en particulier été employé pour le traitement d'un grand nombre de marins qui avaient été brûlés au cours d'une récente collision entre deux bateaux et qui avaient été hospitalisés dans l'hôpital naval de Portsmouth. Toutes les victimes ont été guéries et leurs plaies se sont cicatrisées, sans laisser de trace. Il est néanmoins nécessaire que le traitement par le lait soit commencé immédiatement à l'arrivée des victimes à l'hôpital. 


\section{L'enrichissement en vitamine B12 du gruyère}

Des chercheurs de l'U.S.D.A. ont mis au point un nouveau procédé d'obtention de vitamine $\mathrm{B} 12$ qui consiste à ajouter des bactéries propioniques à des milieux nutritifs fermentescibles contenant du lait écrémé ou du petit-lait.

On sait que ces bactéries sont ajoutées au lait par les fabricants de gruyère, afin de favoriser la formation des trous dans le fromage et de communiquer à ce produit sa saveur caractéristique. En modifiant légèrement le mode opératoire, on est parvenu à augmenter considérablement la quantité de vitamine B12 produite et, alors que le fromage normal renferme 14 à 19 microgrammes de cette vitamine par livre de fromage, le produit fabriqué par le nouveau procédé en contient 57 microgrammes.

On ne connaît pas encore le rôle exact des vitamines B12 dans la nutrition humaine; les recherches entreprises ont montré toutefois qu'elles favorisent l'utilisation des protéines par l'organisme.

\section{Nouveau lait enrichi}

La Borden Co: vient de mettre sur le marché un nouveau lait enrichi qui est livré dans des bouteilles en verre foncé ou dans des récipients cartonnés. La teneur en graisse de ce produit est réglée à $3,8 \%$ et un litre environ de ce lait assure tous les besoins d'un homme adulte en vitamines $\mathrm{A}$ et $\mathrm{D}$, en thiamine, en riboflavine, en niacine, en calcium, en phosphore et en iode, alors que par exemple le lait normal ordinaire ne renferme pas tous ces constituants en quantité suffisante.

\section{Le rôle du lait dans la nourriture des femmes enceintes}

Les biologistes du National Dairy Council ont démontré récemment le rôle essentiel que joue le lait dans la nourriture des futures mères. Ce produit en effet fournit à la femme les quantités suffisantes de calcium, de phosphore et de vitamine D qui sont indispensables à la conservation de la santé de la mère et au développement de l'enfant. Un litre de lait par jour dans les premiers mois de la grossesse et un litre et demi plus tard assurent les quantités de vitamine $\mathrm{D}$, indispensables au phénomène dé calcification. Au contraire, l'addition à la nourriture de concentrés de calcium ne joue pas du tout le même rôle, car en outre le lait fournit les graisses, les protéines, le phosphore et le magnésium, ainsi que d'autres substances encore inconnues qui agissent sur la façon dont le calcium est retenu par l'organisme de la mère et utilisé pour le développement du squelette et des dents de l'enfant. 


\section{Variation de la teneur en résidu sec du lait sur une période de onze années}

Le prix du lait devrait être fonction de la teneur de ce produit en graisse et en résidu sec. Cependant, cette relation désirable n'est pas tonjours réalisée et le tableau ci-dessous qui montre l'évolution du prix de 100 pounds (1) de lait à 3,5\% de matière grasse au cours des onze dernières années indique la valeur relative de la graisse ou du résidu sec contenu dans ce lait d'après les cours en vigueur pendant les années passées en revue :

\begin{tabular}{|c|c|c|c|}
\hline \multirow[t]{2}{*}{ Année } & \multirow{2}{*}{$\begin{array}{l}\text { Valeur du lait } \\
\text { (en dollars) }\end{array}$} & \multicolumn{2}{|c|}{$\begin{array}{l}\text { Pourcentage de cette valeur } \\
\text { représentée par }\end{array}$} \\
\hline & & Matière grasse & Extraitsec dég. \\
\hline $1941 \ldots \ldots \ldots \ldots$ & 2,19 & 56 & 44 \\
\hline $1942 \ldots \ldots \ldots \ldots$ & 2,61 & $\tilde{5} 6$ & 44 \\
\hline $1943 \ldots \ldots \ldots \ldots$ & 2,78 & 58 & 42 \\
\hline $1944 \ldots \ldots \ldots \ldots$ & 2,81 & 57 & 43 \\
\hline $1945 \ldots \ldots \ldots \ldots$ & 2,76 & 58 & 42 \\
\hline $1946 \ldots \ldots \ldots \ldots$ & 3,43 & 64 & 36 \\
\hline $1947 \ldots \ldots \ldots \ldots \ldots$ & 3,34 & 74 & 26 \\
\hline $1948 \ldots \ldots \ldots \ldots \ldots$ & 3,72 & 67 & 33 \\
\hline $1949 \ldots \ldots \ldots \ldots$ & 3,10 & 68 & 32 \\
\hline $1950 \ldots \ldots \ldots \ldots$ & 3,13 & 69 & 31 \\
\hline $1951 \ldots \ldots \ldots \ldots$ & 3,61 & 67 & 33 \\
\hline
\end{tabular}

On voit que depuis 1947 , les matières sèches dégraissées du lait représentent dans le prix du produit un pourcentage qui tend à s'élever.

\section{L'emploi de l'eau oxygénée dans la conservation du lait}

L'emploi de l'eau oxygénée pour assurer la conservation du lait n'est pas une chose nouvelle en soi ; on sait que l'addition d'eau oxygénée à du lait permet de conserver celui-ci pendant un temps assez long, sans qu'il soit nécessaire de le traiter par la chaleur. Néanmoins, on estime qu'avant que ce produit puisse être utilisé, il faudra de longues années, afin de faire la preuve qu'il n'a aucune action sur la santé des consommateurs. L'eau oxygénée risque en effet de détruire les vitamines ou de modifier les autres constituants du lait en altérant ses qualités nutritives et peut-être même en faisant apparaître dans le lait des substances toxiques.

(1) 1 pound $=453,59$ grammes. 


\section{Un nouveau procédé de mise en conserve du lait.}

La Golden State Co. vient de déposer un brevet pour un nouveau procédé de préparation de conserves de lait, Le procédé consiste à soumettre à une stérilisation instantanée le lait par chauffage à très haute température pendant un temps très court. On poursuit ensuite la stérilisation à plus basse température et le lait est refroidi. Par ailleurs, les boîtes et les couvercles de celles-ci sont stérilisés par la vapeur surchauffée. On remplit les boîtes stériles au moyen du lait stérilisé et refroidi et on procède à la fermeture des boîtes dans une atmosphère de vapeur surchauffée dans des conditions aseptiques.

Le nouveau procédé consiste done essentiellement à stériliser séparément le lait et les boîtes et à assurer la mise en boîte dans des conditions stériles.

\section{Le lait traité par les composés fluorés}

On sait qu'on envisage aux Etats-Unis le traitement des eaux d'alimentation par les fluorures, afin de lutter contre les maladies dentaires. Ce traitement présente certaines difficultés et on a envisagé d'ajouter au lait une certaine proportion de fluorures, car la quantité normale de fluorures contenus dans le lait est insuffisante. Il reste à démontrer que l'addition de fluorures ne modifie en rien la valeur nutritive du lait et ses qualités dégustatives. Des recherches sont entreprises dans de nombreux établissements scientifiques, mais dès à présent, certains expérimentateurs ont procédé d'eux-mêmes à l'addition de fluorures au lait consommé dans leur famille et ont enregistré des résultats satisfaisants. L'addition se fait sous la forme de fluorure de sodium dans la proportion de 0,5 partie de fluorures par million de parties de lait.

\section{La vente du lait directement à la consommation}

Les statistiques récentes montrent que les ventes du lait à la consommation continuent à augmenter et représentent aujourd'hui plus de la moitié des 56 milliards de litres de lait produits aux Etats-Unis. La valeur de la production annnuelle de lait et des produits laitiers est estimée à 8 milliards de dollars et en 1950 , ces produits ont représenté 14\% des revenus des fermiers.

Les principaux Etats producteurs restent le Wisconsin, l'Etat de New-York, le Minnesota, la Californie, l'Iowa, la Pensylvanie et le Michigan. Le classement n'est pas tout à fait le même pour la production de beurre. 